\title{
Family Conflict as a Mediator of Caregiver Strain*
}

\author{
Andrew Scharlach
}

Wei Li

\author{
Tapashi B. Dalvi**
}

\begin{abstract}
The present study used structural equation modeling to examine the potential mediating effect of family conflict on caregiver strain in a randomly drawn household sample of 650 adults with primary care responsibility for an adult age 50 or older with a mental disability. Caregiver strain was directly influenced by the conflict, disagreements, and hardships experienced by the caregiver's family. Specifically, family conflict was found to mediate the impact of care recipient mental impairment and caregiver educational level on caregiver strain, and mediate partially the impact of caregiver income and caregiver-care recipient relationship. Findings suggest the importance of considering family-centered approaches when designing interventions to assist family caregivers.
\end{abstract}

$\underline{\text { Key Words: }}$ caregiver distress, elder care, family caregiving, family stress.

Most research on the impact of cognitive impairment has focused on the distress experienced by the primary caregiver (Ory, Hoffman, Yee, Tennstedt, \& Schulz, 1999; Pruchno \& Postashnik, 1998; Schulz, O’Brien, Bookwala, \& Fleissner, 1995). However, there is substantial evidence that an elderly individual's mental impairment can affect other family members, as well, potentially creating stress for the entire family system (Fisher \& Lieberman, 1994; Garwick, Detzner, \& Boss, 1994; Peters-Davis, Moss, \& Pruchno, 1999). Although there frequently is one family member who assumes primary responsibility for providing hands-on care, other family members often provide substantial secondary tangible assistance and social and emotional support (Gaugler, Mendiondo, Smith, \& Schmitt, 2003; Penrod, Kane, Kane, \& Finch, 1995; Tennstedt, McKinlay, \& Sullivan, 1989) and may experience secondary threats to their psychological well-being, physical health, and interactions with other family members (Amirkhanyan \& Wolf, 2003; Brody, Hoffman, Kleban, \& Schoonover, 1989; PetersDavis et al., 1999). The adaptations required by these role shifts and disruptions in interpersonal relationships can undermine family stability, potentially resulting in family conflict and other signs of family stress.

Because families are an important source of social and emotional support to their members, as well as a potential source of stress, disruptions to family functioning can have deleterious effects for individual family members who have demanding care responsibilities such as caring for an elder with mental impairments. Families disrupted by conflict or other signs of family dysfunction are apt to provide less assistance to members with cognitive disabilities (Lieberman \& Fisher, 1999; Matthews \& Rosner, 1988) and less social and emotional support to those family members who have primary care responsibilities (Gaugler et al., 2003; Weihs, Fisher, \& Baird, 2002), potentially exacerbating caregiver strain. Most previous research on family caregiving has focused solely on a single caregiver and ways in which a care recipient's impairments and care needs affect the caregiver's well-being. This study examines the possibility that family conflict and related family hardships not only exacerbate the strain experienced by the primary caregiver

*This research was conducted with support from the California Department of Aging and the Kleiner Family Foundation. The authors are grateful to Teresa Dal Santo for her assistance with all aspects of this project.

**Andrew Scharlach is Kleiner Professor of Aging in the School of Social Welfare at University of California, Berkeley, 120 Haviland, Berkeley, CA 94720-7400 (Scharlach@berkeley.edu). Wei Li is a Quantitative Research Analyst in the Center for Responsible Lending, 302 W Main Street, Durham, NC 27701 (wei.li@ responsiblelending.org). Tapashi B. Dalvi is a Doctoral Candidate in the Department of Epidemiology at University of California, Berkeley, 140 Warren Street, Berkeley, CA 94720 (tapashi@berkeley.edu). 
but also serve as important mechanisms through which an elderly relative's mental impairment can produce caregiver strain.

\section{Theoretical Framework}

This study draws upon the general stress process model described by Pearlin (Pearlin, Lieberman, Menaghan, \& Mullan, 1981). As applied to family caregiving, this model conceptualizes caregiver stress as a product of four related factors: the caregiving context, primary stressors, secondary stressors, and potential mediators (Pearlin, Mullan, Semple, \& Skaff, 1990). Family conflicts are understood as secondary stressors resulting from the care demands that serve as primary stressors (e.g., cognitive impairment, problematic behaviors). Indeed, Pearlin and colleagues described the family as "a central arena for secondary role strains" (Pearlin et al., 1990, p. 588). Family conflict and other secondary stressors can directly impact caregiver stress, while also mediating the effect of primary stressors. This entire stress process occurs in the context of background demographic characteristics (e.g., gender, socioeconomic status, caregiver-care recipient relationship, living arrangement). Although there is extensive evidence supporting the stress process model, there has been only limited attention to the role of family conflict and its role as a potential mediator of caregiver strain.

Our theoretical understanding of family strains associated with elder care also draws upon a family systems orientation to family stress and adaptation (McCubbin \& McCubbin, 1993). From this perspective, an elder's illness and resulting care activities are seen to occur within the context of uniquely meaningful and continuing family relationships, which include a shared social reality that affects how the elder care situation is understood and accommodated (Kleinman, Eisenberg, \& Good, 1978). A family member's chronic illness or impairment can disrupt existing relationship patterns, potentially requiring adaptations that can result in interpersonal conflict or other signs of family dysfunction (Kuypers \& Bengtson, 1983). In turn, family dysfunction can undermine the family's competence as a supportive entity for its members (Weihs et al., 2002). One result may be increased distress for those family members who have primary caregiving responsibilities.

\section{Family Conflict Associated with Cognitive Impairment}

Mental impairment can contribute directly and indirectly to family stress. Family members who become directly involved in the provision of handson care or supervision are apt to experience personal and interpersonal role strain. Even those not directly involved in providing care may experience deleterious effects on their health and well-being (Lieberman \& Fisher, 1995). Indeed, some studies have found that family members who are not primary caregivers may experience levels of strain that are the same or even greater than those experienced by primary caregivers (Amirkhanyan $\&$ Wolf, 2003; Lieberman \& Fisher, 1995). Within families caring for a relative with Alzheimer's disease, for example, the negative effects of providing care experienced by the spouses of caregiving sons and daughters generally are quite similar to the effects experienced by the sons and daughters themselves (Peters-Davis et al., 1999). The personal and interpersonal stresses associated with caring for a family member with mental impairments are manifested in three types of conflictual family relationships: (a) disagreements regarding the nature of the illness and resultant care needs, (b) disagreements regarding family member's actions or inactions toward the care recipient, (c) disagreements regarding family member's actions or inactions toward the family member with primary care responsibilities (Pearlin et al., 1990; Semple, 1992).

The primary stressors associated with the care recipient's need for assistance are an important predictor of the extent of secondary family stress and conflict. Family functioning in families of braininjured persons has been found to be affected directly by the extent of care recipients' behavioral and cognitive problems (Lieberman \& Fisher, 1999), including their neurobehavioral, neuropsychological, and affective functioning and their "awareness of deficit" (Ergh, Rapport, Coleman, \& Hanks, 2002). The severity of the care recipient's illness and the amount of time devoted to care have been found to impact the health and well-being of all family members, including spouses, offspring, and in-laws (Fisher \& Lieberman, 1994).

Relationship to the family care recipient also seems to be an important factor. Adult sons and daughters, for example, appear more likely than spouses to experience family conflict. In one study of 555 caregivers, $82 \%$ of offspring caregivers reported family conflict compared to only $55 \%$ of 
spousal caregivers (Semple, 1992). Spouses generally experienced conflicts with sons or daughters, whereas offspring were more likely to report conflict with siblings. It is not known to what extent differences in the likelihood of coresidence contribute to these differences between spouses and adult offspring.

Gender, while consistently identified as a factor contributing to caregiver strain (Miller \& Cafasso, 1992; Yee \& Schulz, 2000), has infrequently been examined in relationship to family conflict and stress. In one of the few studies to address gender issues directly, women were found to be more likely than men to report family conflict, and with a broader range of family members (Semple, 1992). There is some evidence that caregiving daughters may receive less help from spouses and siblings than do caregiving sons, although they may also expect less help (Matthews \& Rosner, 1988). Others have argued that women may place more importance on the quality of family relationships, making them more sensitive to family conflict (Mui, 1995).

\section{Families as Sources of Support and Stress}

Families that are able to adapt to the demands of chronic care without excessive conflict or disruption are likely to serve as potential sources of support for the family member who assumes the primary caregiving role for an elderly relative. Daughters caring for parents with disabilities, for example, reported feeling more rewards associated with providing care when their siblings provide emotional support and understanding (Brody et al., 1989). Caregivers' spouses can be an important source of support (Fisher \& Lieberman, 1994; Peters-Davis et al., 1999), and husbands' instrumental support for caregiving wives has been found to be a protective factor for their wives' physical health (Franks \& Stephens, 1996). Family members, however, also can be a source of substantial stress for family caregivers. As many as $40 \%$ of caregivers reportedly experience serious conflict with a family member, usually a sibling unwilling to provide the expected amount of help (Brody et al.; Strawbridge \& Wallhagen, 1991).

Conflictual family relationships can have deleterious impacts on caregivers in a variety of ways. For example, behavioral and cognitive deficits can deplete family resources, undermining family members' ability to provide consistent and reliable assistance to the care recipient and emotional support for the primary caregiver (Anderson, Parmenter, \&
Mok, 2002). Conflicted family relationships also can interfere with caregivers' ability to regulate the emotional distress that frequently accompanies the care situation (Fiscella, Franks, \& Shields, 1997; Weihs et al., 2002). In addition, conflict among family members regarding the appropriate type and amount of support for care recipients can violate caregivers' normative expectations, arousing caregivers' anger and undermining the caregivers' sense of competence, contributing to negative self-evaluations and depression (Semple, 1992).

Relatively little empirical research has focused on the impact of family conflict on caregiver well-being. One study of 100 adult child caregivers revealed that family conflict predicted higher burden and poorer mental health, even after controlling for the relationship with care recipient, age, income, gender, and caregiver needs (Strawbridge \& Wallhagen, 1991). Another study of 64 spousal caregivers of patients who had sustained severe traumatic brain injury found high levels of psychological distress among spousal caregivers who reported unhealthy family functioning (Anderson et al., 2002). Finally, a comprehensive study of 555 adults caring for a cognitively impaired spouse or parent associated caregiver anger and depression with particular types of family conflict (Semple, 1992).

To our knowledge, only one study has explored the potential mediating effect of family conflict on caregiver well-being. Deimling, Smerglia, and Schaefer (2001) drew upon Pearlin's model of stress and coping (Pearlin et al., 1990) to examine interrelationships among care recipient impairment, family impact, and caregiver stress among 244 family caregivers. Family conflict and disagreements were found to mediate the association between care recipient impairment and caregiver depressive symptoms. However, generalization is limited by the use of a convenience sample composed primarily of service users, more than $80 \%$ of whom were White.

The present study expands upon previous research by using structural equation modeling to examine the extent to which the impact of the caregiving situation on the primary caregiver is mediated by family conflict. Unlike previous studies, this study utilized population-based sampling methods to obtain a large, highly representative, racially and ethnically diverse sample of family caregivers. The conceptual model guiding this investigation is displayed in Figure 1. In accordance with Pearlin's caregiver stress paradigm (Pearlin et al., 1990), 


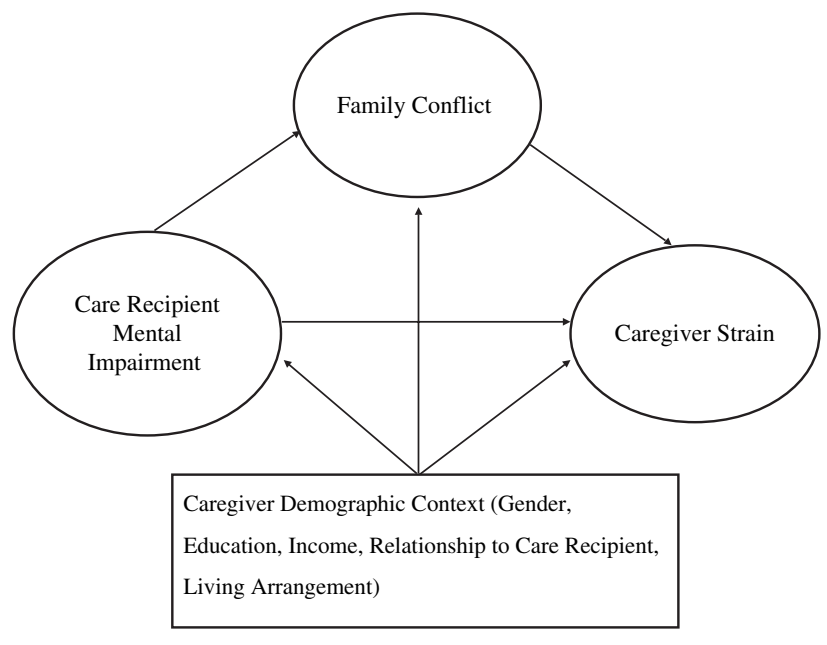

Figure 1. Hypothesized Model.

caregiver strain, the focal construct in the model, was hypothesized as being influenced directly and indirectly by primary stressors (care recipient's mental impairment), secondary stressors (family distress), and contextual demographic characteristics (caregiver gender, education, income, relationship to the care recipient, and living arrangement). These demographic variables are added into the model as exogenous manifest variables.

\section{Methods}

\section{Procedures}

A random sample of 650 caregivers participated in a $30 \mathrm{~min}$ telephone interview between March and August 2002. The initial statewide sampling frame consisted of a list of telephone numbers reflective of households with phones throughout California, which was sorted into working blocks of 100 contiguous numbers, then randomly sampled within working blocks. To ensure that some unlisted phone numbers were included in the sample, the original list was supplemented by using a working number as a seed number from which one other number was generated by adding a constant. Using computer-assisted telephone interviewing equipment and software, interviews were conducted in English and Spanish.

\section{Sample}

Respondents were asked whether they, or someone else in their household, "currently provide assistance or support to an adult relative or friend [age 50 or older] who is ill, disabled, or elderly." "Assistance or support" was defined as providing assistance with personal needs, household chores, taking care of finances, or arranging for outside services. Of 7,999 respondents who agreed to participate in the survey, 1,643 indicated that they currently met our criteria and completed the remainder of the survey. Of these, 650 had primary responsibility for providing care to a family member and serve as the sample for the analyses reported here.

Respondents had a median age of 51 years old, $75 \%$ were female, $61 \%$ were married, $68 \%$ had some post-high school education, and $60 \%$ had household incomes over \$30,000. Most (57\%) were White/Caucasian, 27\% were Hispanic/Latino, 6\% were Black/African American, and 6\% were Asian or Pacific Islander; $85 \%$ were born in the United States and $7 \%$ were born in Mexico. This demographic profile is generally consistent with findings from a 1996 national study (National Alliance for Caregiving \& the American Association of Retired Persons, 1997), adjusting for differences between California's population and that of the entire United States.

Sixty-eight percent of the care recipients were parents of respondents, $16 \%$ were spouses, and 16\% were other relatives, including grandparents, siblings, and cousins. The median age of care recipients was 77 ; most were female (69\%), $49 \%$ were widowed, and tended to live either with the caregiver $(47 \%)$ or alone in their own home $(31 \%)$. Sixty-one percent of the care recipients were White/Caucasian, 25\% were Hispanic/Latino, 7\% were Black/African American, and 6\% were Asian or Pacific Islander; $76 \%$ were born in the United States, and $12 \%$ were born in Mexico.

\section{Measures}

The telephone survey elicited information regarding the following domains: (a) background demographic characteristics, (b) care recipient mental impairment, (c) perceived family conflicts, and (d) caregiver strain.

Background demographic variables included caregiver gender, education (treated as six ordered categorical variables, from "less than high school" to "postgraduate degree"), household income (treated as seven ordered categorical variables, from "under $\$ 10,000$ " to "over $\$ 80,000$ "), relationship to the care recipient (spouse vs. nonspouse), and living arrangement (together with care recipient vs. separate). 
Care receiver mental disability was assessed by asking respondents to indicate whether the care receiver had the following conditions: (a) severe memory problems or dementia (such as asking the same question over and over, forgetting what day it is, or losing or misplacing things), (b) behavior problems (such as arguing, being irritable, verbally aggressive, or waking you or other family members up at night), or (c) mental health problems or emotional problems (such as appearing anxious or worried, sad or depressed, or having crying spells). Behavior and mental health problems were included in our measure of mental disability because of the repeated finding that behavior problems are more damaging to caregiver well-being than are cognitive impairments alone (Schulz \& Williamson, 1991; Schulz et al., 1995). Respondents indicated that $36 \%$ of their care recipients had severe memory problems, 22\% had behavior problems, and 34\% had mental health problems.

Family conflict was assessed by asking respondents to indicate, on a four-point Likert scale (from $1=$ none at all to $4=$ very $m u c h)$, the extent to which the care receiver's need for care had (a) created conflict or disagreements in their family or (b) been a hardship for their family. Family conflict or disagreements were reported by $49 \%$ of respondents, and family hardship was reported by $58 \%$.

Caregiver strain was assessed by asking respondents to indicate, on a five-point Likert scale (from $1=$ none at all to $5=$ a great deal), the extent to which caring for the care recipient resulted in the following: (a) financial hardship, (b) physical strain, or (c) emotional stress. Financial hardship resulting from providing care was reported by $49 \%$ of respondents, physical strain was reported by $62 \%$, and emotional stress by $76 \%$.

\section{Analyses}

Structural equation modeling was used to test the hypothesized model using the SAS Covariance Analysis of Linear Structural Equations (CALIS) procedure (SAS Institute, 1999). In our conceptual model (see Figure 1), caregiver strain and family conflict were indicated by ordinal variables, care receiver mental impairment was indicated by binary variables, and the demographic context was indicated by a combination of ordinal and binary variables. Because of possible deviations from multivariate normality, weighted least squares methods were employed to obtain asymptotically distribution-free estimates of the parameters and their standard errors (Browne \& Cudeck, 1993). A covariance matrix was calculated in order to examine associations among the observed variables (see Table 1). The inverse matrix of the estimated asymptotic covariances of the sample covariance matrix was used as the weight matrix. The weight matrix was obtained by using Browne's method (Browne \& Cudeck).

The first step in the analysis was to fit a "full" model to the data by including all possible linkages proposed in the hypothesized model (see Figure 1). The second step was to fit a final model to the data by eliminating all statistically insignificant linkages from the full model. To assess the fit between the theoretical model and the actual data, we calculated the chi-square test of significance, the root mean squared error of approximation (RMSEA), and Bentler's comparative fit index (CFI). To test the significance of a mediating effect, we used Sobel's methods (Sobel, 1982, 1986).

\section{Results}

We first fit a structural equation model to the sample covariance by including all possible linkages proposed in our full hypothesized model (Figure 1). Initial analyses identified 14 linkages that were not statistically significant at the .05 probability level. Fit statistics of this initial model were as follows: $\chi^{2}=61.37(d f=39, N=650, p=.01)$, CFI $=$ 0.975 , RMSEA $=0.030(0.014,0.043)$. By eliminating the 14 insignificant linkages from the full model, we were able to produce a final model with a much better fit to the data: $\chi^{2}=50.96(d f=43$, $N=650, p=.19)$, CFI $=0.990$, RMSEA $=0.017$ $(0.000,0.033)$. The final model fit statistics were considered quite acceptable given the sample size and the asymptotically distribution-free estimation method used in these analyses.

The measures of the structural equation model fit and the estimated model parameters, including direct and indirect effects, are presented in Table 2. Standardized parameter estimates are displayed in Figure 2. As hypothesized, care recipient mental impairment and family conflict were each found to have a positive total effect on caregiver strain. The effect of care recipient mental impairment on caregiver strain was fully mediated by perceived family conflict. 


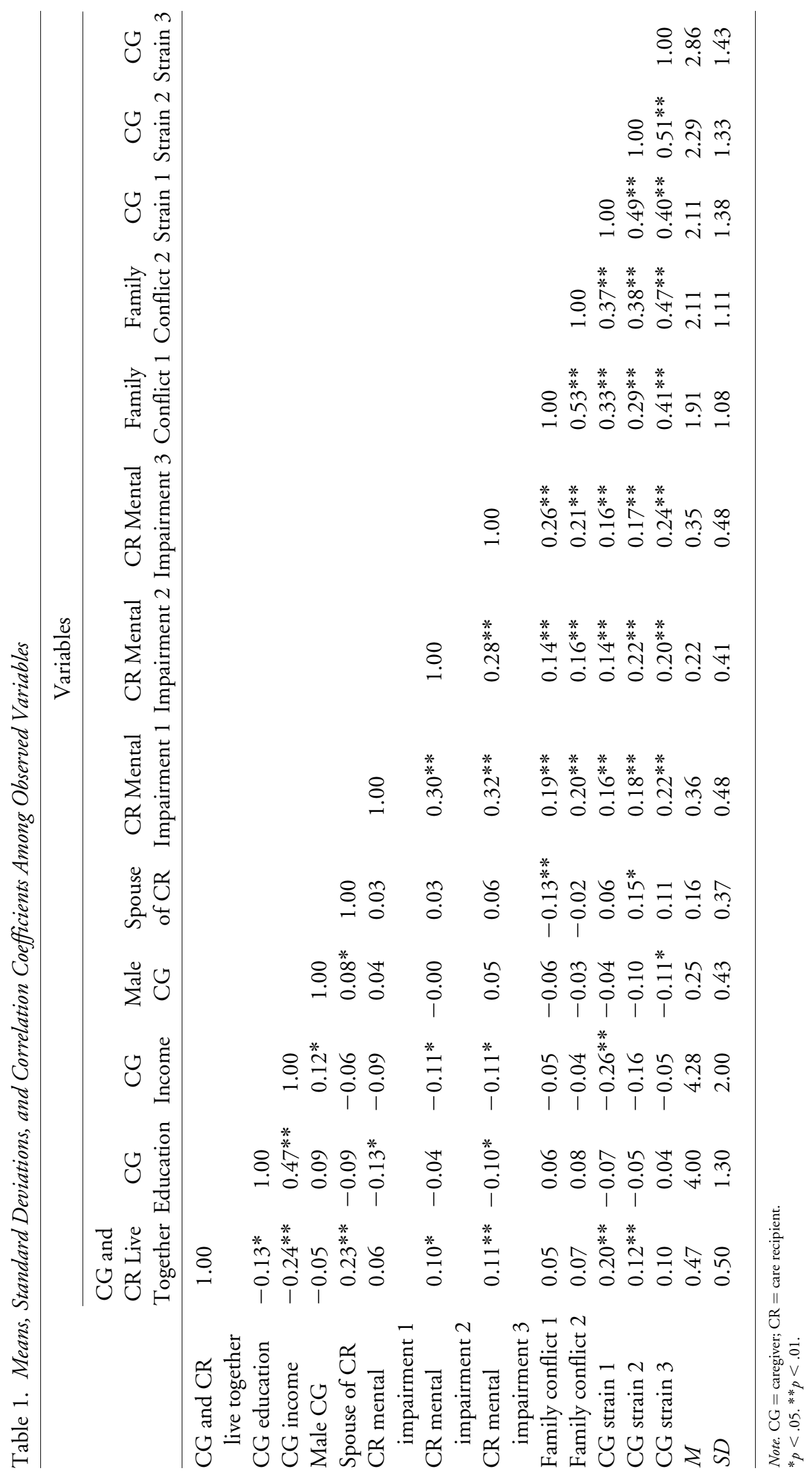


Table 2. Unstandardized Path Coefficients for Final Structural Equation Model

\begin{tabular}{|c|c|c|c|}
\hline \multirow{2}{*}{$\begin{array}{l}\text { Independent Constructs } \\
\text { by Effect Type }\end{array}$} & \multicolumn{3}{|c|}{ Dependent Constructs } \\
\hline & CR Mental Impairment & Family Conflict & CG Strain \\
\hline \multicolumn{4}{|l|}{ Direct effects } \\
\hline CG-CR live together & $0.06^{*}$ & $-^{\mathrm{a}}$ & $-^{\mathrm{a}}$ \\
\hline CG education & $-^{\mathrm{a}}$ & $0.07 *$ & $-^{\mathrm{a}}$ \\
\hline CG income & $-0.02 *$ & $-^{a}$ & $-0.09^{*}$ \\
\hline Spouse of CR & $-^{a}$ & $-0.52^{*}$ & $0.95^{*}$ \\
\hline CG gender & $-^{\mathrm{a}}$ & $-^{\mathrm{a}}$ & $-0.34^{*}$ \\
\hline CR mental impairment & ${ }^{\mathrm{b}}$ & $1.99 *$ & - $^{\mathrm{a}}$ \\
\hline Family conflict & $-^{\mathrm{b}}$ & $-^{\mathrm{b}}$ & $1.12^{*}$ \\
\hline \multicolumn{4}{|l|}{ Indirect effects } \\
\hline CG-CR living together & & $0.12^{*}$ & $0.14^{*}$ \\
\hline CG education & & $-^{a}$ & $0.08^{*}$ \\
\hline CG income & & $-0.04^{*}$ & $-0.05^{*}$ \\
\hline Spouse of CR & $-^{\mathrm{b}}$ & $-^{a}$ & $-0.58^{*}$ \\
\hline CG gender & & $-^{\mathrm{a}}$ & - $^{\mathrm{a}}$ \\
\hline CR mental impairment & & $-^{\mathrm{b}}$ & $2.22 *$ \\
\hline Family conflict & & $-^{b}$ & $-\mathrm{b}$ \\
\hline \multicolumn{4}{|l|}{ Total effects } \\
\hline CG-CR live together & $0.06^{*}$ & $0.12^{*}$ & $0.16^{*}$ \\
\hline CG education & $-^{a}$ & $0.07^{*}$ & $0.08^{*}$ \\
\hline CG income & $-0.02 *$ & $-0.04^{*}$ & $-0.14^{*}$ \\
\hline Spouse of CR & $-^{a}$ & $-0.52^{*}$ & $0.38^{*}$ \\
\hline CG gender & $-^{\mathrm{a}}$ & $-^{a}$ & $-0.34^{*}$ \\
\hline CR mental impairment & $-^{\mathrm{b}}$ & $1.99^{*}$ & $2.22 *$ \\
\hline Family conflict & $-^{b}$ & ${ }^{\mathrm{b}}$ & $1.12^{*}$ \\
\hline
\end{tabular}

Note. $\mathrm{CG}=$ caregiver; $\mathrm{CR}=$ care recipient. Model fit statistics: $\chi^{2}=50.96(d f=43, N=650, p=.19)$, comparative fit index $=0.990$, root mean squared error of approximation $=0.017(0.000,0.033)$. Indirect effects were calculated by multiplying the coefficients associated with the path of influence from the independent construct to the dependent construct (see Figure 2). For example, CR mental impairment has an indirect effect on CG strain via its direct influence on family conflict $(2.224=1.990 \times 1.118)$.

a Linkages that were included in the original hypothesized model but found not to be statistically significant at the .05 probability level; these linkages were eliminated from the final

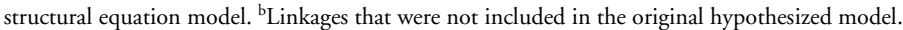
${ }^{*} p<.05$.

Living together, greater caregiver education, lower caregiver household income, caring for a spouse, and being female were found to have total effects on higher caregiver strain. The effect of caregiver income on caregiver strain was partially mediated $(33 \%)$ by care recipient mental impairment and family conflict; the effect of caring for a spouse was partially mediated (38\%) by family conflict; the effect of caregiver education was fully mediated by family conflict; and the effect of living together was fully mediated by care recipient mental impairment. Caregiver gender only had a direct effect on caregiver strain.

Living together, greater caregiver education, lower caregiver household income, and caring for someone other than a spouse each had a total effect on higher family conflict. The effects of caregiver income and living together on family conflict were fully mediated by care recipient mental impairment. Caregiver education and caring for a spouse each had only a direct effect on family conflict. Living together and lower caregiver income both had a total effect on higher care recipient mental impairment.

\section{Discussion}

The findings reported here provide evidence that caregiver strain is directly influenced by the conflict, disagreements, and hardships experienced by the caregiver's family. Specifically, family conflict was found to mediate the impact of care recipient mental impairment on caregiver strain. 


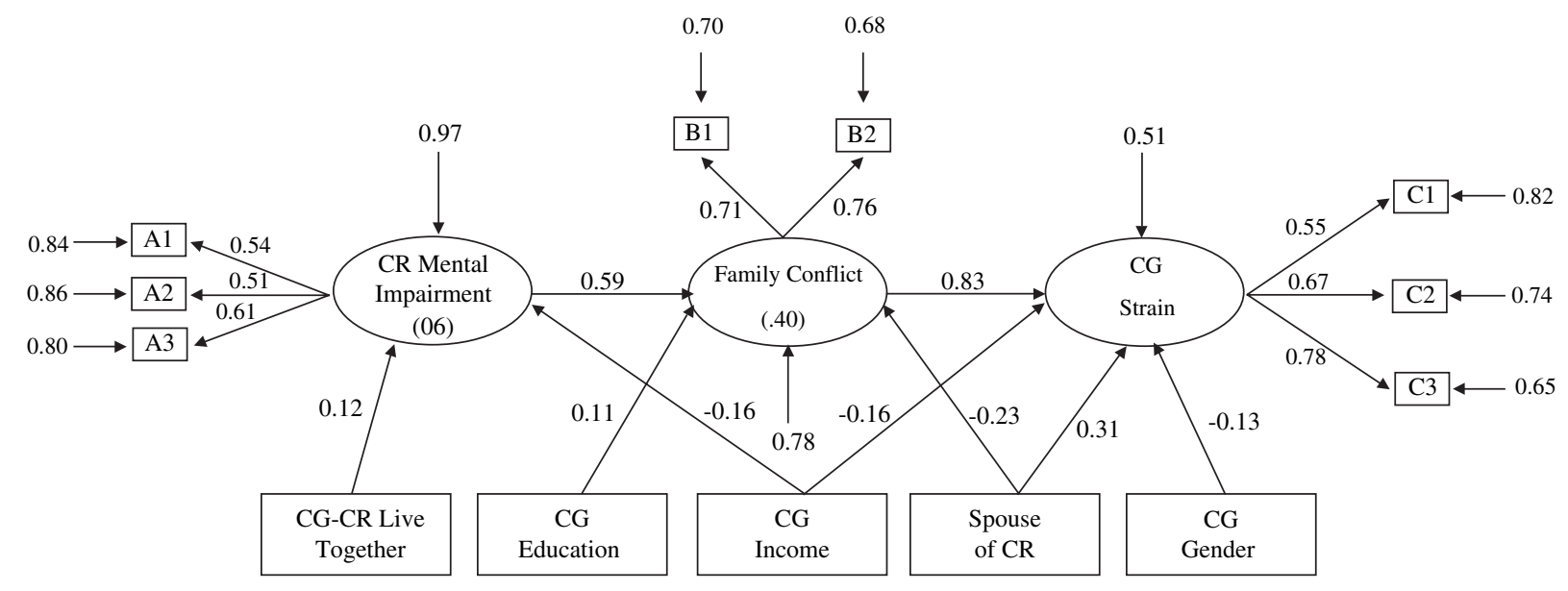

Figure 2. Final Structural Equation Model of Care Recipient Mental Impairment, Family Conflict, and Caregiver Strain. Note. All path coefficients are statistically significant at the .05 probability level. Standardized parameter estimates ( $R^{2}$ in parentheses); $N=650$.

There are several possible ways in which family conflict may affect caregiver well-being. First, family conflict is apt to be manifested in disagreements and stressful interactions involving the primary caregiver (Lieberman \& Fisher, 1999; Semple, 1992; Strawbridge \& Wallhagen, 1991), which would add to the caregiver's strain. Semple's study of caregivers of relatives with Alzheimer's disease, for example, found that conflict involving family members' actions or attitudes toward the caregiver can exacerbate depressive symptoms. The negative effect of cognitive impairment on caregivers may be due in part to its disruption of normal family environment and decision-making processes (Deimling et al., 2001) and lack of consensus among family members regarding the care situation (Pruchno, Burant, \& Peters, 1997). Second, because conflicted families generally provide less care (Lieberman \& Fisher, 1999), caregivers may receive less instrumental or emotional support with care provision or both (Brody et al., 1989; Gaugler et al., 2003; Penrod et al., 1995), adding to their physical, financial, or emotional strain. Behavioral and cognitive problems can deplete family resources, thus leading to higher distress in caregivers (Anderson et al., 2002). Third, it is possible that, in assessing his or her own level of strain, a caregiver may take into account evidence of stress or anger among family members (Deimling et al.; Lieberman \& Fisher, 1995). For example, in Semple's study, family anger and attitudes toward the care recipient were associated with feelings of anger in the primary caregiver. In their study of dementia patients and their family caregivers, Lieberman and Fisher concluded that sons and daughters who perceive their parent as more stressed, on the basis of his or her use of services, experience reduced psychological well-being.

It is notable that care recipient mental impairment did not have a significant direct effect on caregiver strain, but rather was fully mediated by family conflict. This finding suggests that the physical and psychological distress commonly found among persons caring for a family member with mental impairments may be due in part to the disruption experienced by the family system and other secondary contexts. Among the secondary stressors apt to be associated with a family member's mental impairment are disrupted family decision making (Deimling et al., 2001), conflict over appropriate family role behavior (Semple, 1992), restimulation of prior interpersonal conflicts (Kuypers \& Bengtson, 1983), disparate perceptions of the impaired relative's psychological status (Boss \& Kaplan, 2004), conflict with other roles and responsibilities (FredriksenGoldsen \& Scharlach, 2001), and economic and social hardships (Pearlin et al., 1990). In accordance with Pearlin's stress process model (Pearlin et al.), our findings suggest that secondary stressors such as these may serve as an important mechanism by which care recipients' mental impairment exacerbates the strain experienced by family members who have primary responsibility for their care.

Caregiver strain associated with caregiver income, education, and care recipient relationship also was mediated in large part by family conflict. Caregivers with lower household incomes are apt to have fewer actual and potential support options for themselves and their care recipients. They are less likely to 
utilize paid care providers, therefore potentially increasing the pressure on other family members to assist with care provision and creating opportunities for disagreements regarding the amount and adequacy of care provision (Deimling et al., 2001; Kuypers \& Bengtson, 1983). Caregivers with lower household incomes also are less likely to utilize counseling, respite care, and other social and emotional support services for themselves (Rodriguez, Kosloski, \& Montgomery, 2003), potentially increasing their reliance on family members for social and emotional support and increasing their sensitivity to disagreements, interpersonal slights, and other signs of family conflict.

Caregivers with higher levels of formal education experienced greater family conflict, which mediated fully the association of education with caregiver strain. It seems likely that more highly educated caregivers will have higher expectations of other family members and of themselves, potentially contributing to disagreements over care-related norms and behavior; they also may have jobs that are more psychologically stressful. In addition, interpersonal and interactional disruptions associated with a family member's cognitive impairment may be particularly distressing for more highly educated caregivers.

Spouses reported greater caregiver strain, whereas sons and daughters experienced greater family conflict. Higher distress levels are commonly found among individuals caring for a cognitively impaired spouse (Deimling et al., 2001; Yee \& Schulz, 2000). In addition to the day-to-day demands of caregiving, spouses whose partner has mental impairments are especially vulnerable to social isolation, role captivity, and the loss of a primary source of social and psychological well-being (Hooker, ManoogianO’Dell, Monahan, Frazier, \& Shifren, 2000). Individuals caring for a parent, on the other hand, are apt to experience disagreements with siblings over perceived inadequacies in the amount and type of parent care provided, insufficient social or emotional support for the caregiver, and other actual or perceived inequities related in part to the lack of clear societal norms regarding appropriate parent care (Ingersoll-Dayton, Neal, Ha, \& Hammer, 2003; Semple, 1992).

Gender maintained a direct contribution to caregiver strain. This finding is consistent with the large body of research that found that women experienced higher levels of caregiver strain, even after controlling for other factors (Miller \& Cafasso, 1992; Yee \&
Schulz, 2000). Women not only take on more intensive care responsibilities but also may become more emotionally invested in the care process, making them more vulnerable to negative affective responses to emotionally difficult care situations such as those involving care for a family member with mental impairments (Miller, 1990). Gender differences in self-reported caregiving-related strain also may reflect women's greater tendency to express emotional distress and other negative affects (Schulz \& Williamson, 1991).

\section{Implications for Practice}

Findings from this study suggest that familycentered intervention approaches may have particular utility for alleviating distress among caregivers for relatives who have mental impairments. Familycentered approaches generally attempt to do one or more of the following: (a) improve collaboration and coordination among family members, (b) facilitate reorganization of family roles and adjustment of expectations and norms, (c) enhance family closeness and mutually supportive interactions, (d) minimize interpersonal hostility and conflict, or (e) mobilize the family's natural support systems (Weihs et al., 2002). Such approaches contrast with most existing caregiver support programs and services that are focused on the needs of individual caregivers rather than the family system. Familycentered caregiver support services may be especially beneficial for non-White and Hispanic caregiving families, where family well-being may take precedence over caregiver well-being as a motivator for caregiving activities and service use (Scharlach, Kellam, Ong, Goldstein, \& Baskin, 2006).

Weihs et al. (2002) have identified three types of family-focused interventions that practitioners may find especially useful with families caring for chronically impaired members. First, psychoeducational interventions can help to improve family members' understanding of disease processes and their management, primarily through education and behavior change. Second, family relationship interventions can help to foster improved family functioning through counseling that enhances relationship quality, communication, collaboration, and conflict resolution. Families with more organized decisionmaking processes, for example, are able to provide more assistance to cognitively impaired members, and do so with less individual or family strain 
(Deimling et al., 2001; Lieberman \& Fisher, 1999). Third, family therapy can be used to help in reducing the impact and duration of residual disease complications and comorbid psychiatric disorders. There have been relatively few clinical trials or other examples of empirical evidence regarding the effectiveness of family-centered intervention approaches such as these with families caring for adults with mental impairment or other chronic diseases. Moreover, most clinical studies have combined a number of intervention approaches, making it difficult to attribute effectiveness to a particular modality. Thus, future studies assessing the potential beneficial effects of family-based interventions with caregiving families, and the mechanisms through which those effects are obtained, clearly are warranted.

\section{Implications for Research and Theoretical Development}

This study provides supportive evidence for the utility of a family systems approach to understanding elder care and its effects on primary caregivers. These findings are based on data from a large, racially, and ethnically diverse sample drawn using population-based sampling methods, unlike previous studies that have relied on convenience samples and have had little racial or ethnic diversity. Even this study, however, had insufficient non-White and Hispanic respondents to be able to examine the culturally mediated processes guiding families' responses to chronic illness. The present study also is limited by the small number of questions pertaining to the family impact of caregiving and reliance on single-item indicators for some other latent constructs. The cross-sectional design undoubtedly understates complex interpersonal processes underlying family conflict and precludes inferences regarding causation or the sources of particular types of family conflict. Reciprocal paths cannot be ruled out; for example, more highly distressed caregivers may be more dissatisfied with the support they receive from other family members, potentially contributing to the perception or actuality of family conflict. In addition, the results presented here pertain to the caregiver's perception rather than actual evidence of family conflict, suggesting the need for future research collecting clinical and observational data regarding interactional processes within caregiving families. Finally, the "mentally impaired" group in this study likely included many care recipients with relatively mild levels of cognitive impairment, in light of the population-based nature of the sample and global assessment measures utilized here. Given the strong association commonly found between level of mental impairment and caregiver distress (Lieberman \& Fisher, 1999; Smerglia \& Deimling, 1997), it is possible that our findings understate the direct effects of mental impairment on caregiver strain and overstate the mediating role of family conflict.

Additional research is needed regarding the factors that may make families especially vulnerable to conflict and dysfunction when confronted with demanding care situations. Preexisting family dynamics may be an especially important area of inquiry. Lieberman and Fisher (1999), for example, found that the health and well-being of adults whose parent has Alzheimer's disease were directly associated with perceived attributes of family functioning, such as family world view, family structure and organization, and family emotional management. Also needed is research regarding ways in which family conflict may impact the care recipient. There is some evidence, for example, the families that experience more conflict associated with caregiving provide significantly less help than families that experience less conflict (Lieberman \& Fisher, 1999).

The findings reported here contribute to existing knowledge regarding family caregiving by providing new evidence regarding the potential mediating effect of family conflict on caregiver well-being. These findings also support the need for increased attention to family-centered intervention approaches designed to reduce conflict in caregiving families and enhance the well-being of caregiving family members. Needed now is further research examining the family context within which caregiving occurs, the impacts of caregiving on family systems and their members, and the interventions that can improve family functioning.

\section{References}

Amirkhanyan, A. A., \& Wolf, D. A. (2003). Caregiver stress and noncaregiver stress: Exploring the pathways of psychiatric morbidity. The Gerontologist, 43, 817-827.

Anderson, M. I., Parmenter, T. R., \& Mok, M. (2002). The relationship between neurobehavioural problems of severe traumatic brain injury (TBI), family functioning and the psychological well-being of the spouse/caregiver: Path model analysis. Brain Injury, 16, 743-757.

Boss, P., \& Kaplan, L. (2004). Ambiguous loss and ambivalence when a parent had dementia. Contemporary Perspectives in Family Research, 4, 207-224. 
Brody, E. M., Hoffman, C., Kleban, M. H., \& Schoonover, C. B. (1989). Caregiving daughters and their local siblings: Perceptions, strains, and interactions. The Gerontologist, 29, 529-538.

Browne, M., \& Cudeck, R. (1993). Alternative ways of assessing model fit. In K. Bollen \& J. S. Long (Eds.), Testing structural equation models (pp. 136-162). Newbury Park, CA: Sage Publications.

Deimling, G. T., Smerglia, V. L., \& Schaefer, M. L. (2001). The impact of family environment and decision-making satisfaction on caregiver depression: A path analytic model. Journal of Aging and Health, 13, 47-71.

Ergh, T. C., Rapport, L. J., Coleman, R. D., \& Hanks, R. A. (2002). Predictors of caregiver and family functioning following traumatic brain injury: Social support moderates caregiver distress. Journal of Head Trauma Rehabilitation, 17, 155-174.

Fiscella, K., Franks, P., \& Shields, C. (1997). Perceived family criticism and primary care utilization: Psychosocial and biomedical pathways. Family Process, 36, 25-41.

Fisher, L., \& Lieberman, M. A. (1994). Alzheimer's disease: The impact of the family on spouses, offspring, and inlaws. Family Process, 33, 305-325.

Franks, M. M., \& Stephens, A. P. (1996). Social support in the context of caregiving: Husbands' provision of support to wives involved in parent care. Journals of Gerontology B, Psychological Sciences and Social Sciences, 51, 43-52.

Fredriksen-Goldsen, K. I., \& Scharlach, A. E. (2001). Families and work: New directions in the twenty-first century. New York: Oxford University Press.

Garwick, A. W., Detzner, D., \& Boss, P. (1994). Family perceptions of living with Alzheimer's disease. Family Process, 33, 327-340.

Gaugler, J. E., Mendiondo, M., Smith, C. D., \& Schmitt, F. A. (2003). Secondary dementia caregiving and its consequences. American Journal of Alzheimer's Disease and Other Dementia, 18, 300-308.

Hooker, K., Manoogian-O’Dell, M., Monahan, D., Frazier, L., \& Shifren, K. (2000). Does type of disease matter? Gender differences among Alzheimer's and Parkinson's disease spouse caregivers. The Gerontologist, $40,568-573$.

Ingersoll-Dayton, B., Neal, M., Ha, J., \& Hammer, L. (2003). Redressing inequity in parent care among siblings. Journal of Marriage and Family, 65, 201-212.

Kleinman, A., Eisenberg, L., \& Good, B. (1978). Culture, illness, and care: Clinical lessons from anthropologic and cross-cultural research. Annals of Internal Medicine, 88, 251-258.

Kuypers, J., \& Bengtson, V. (1983). Toward competence in the older family. In T. Brubaker (Ed.), Family relations in later life (pp. 211-228). Beverly Hills: Sage.

Lieberman, M. A., \& Fisher, L. (1995). The impact of chronic illness on the health and well-being of family members. The Gerontologist, 35, 94-102.

Lieberman, M. A., \& Fisher, L. (1999). The effects of family conflict resolution and decision making on the provision of help for an elder with Alzheimer's disease. The Gerontologist, 39, 159-166.

Matthews, S., \& Rosner, T. (1988). Shared filial responsibility: The family as the primary caregiver. Journal of Marriage and the Family, 50, $185-195$.

McCubbin, M., \& McCubbin, H. (1993). Family coping with health crises: The resiliency model of family stress, adjustment and adaptation. In C. Danielson, B. Hamel-Bissel, \& P. Winstead-Fry (Eds.), Families, health, and illness (pp. 21-64). St. Louis, MO: Mosby.

Miller, B. (1990). Gender differences in spouse caregiver strain: Socialization and role explanations. Journal of Marriage and the Family, 52, 311-322.

Miller, B., \& Cafasso, L. (1992). Gender differences in caregiving: Fact or artifact? The Gerontologist, 32, 498-507.
Mui, A. (1995). Multidimensional predictors of caregiver strain among older persons caring for frail spouses. Journal of Marriage and the Family, 57, 733-740.

National Alliance for Caregiving \& the American Association of Retired Persons. (1997, June). Family caregiving in the U.S.: Findings from a national survey. Washington, DC: Author.

Ory, M. R., Hoffman, R., Yee, J., Tennstedt, S., \& Schulz, R. (1999). Prevalence and impact of caregiving: A detailed comparison between dementia and nondementia caregivers. The Gerontologist, 39, 177-183.

Pearlin, L. I., Lieberman, M. A., Menaghan, E. G., \& Mullan, J. T. (1981). The stress process. Journal of Health and Social Behavior, 22, 337-356.

Pearlin, L. I., Mullan, J. T., Semple, S., \& Skaff, M. (1990). Caregiving and the stress process: An overview of concepts and their measures. The Gerontologist, 30, 583-594.

Penrod, J. D., Kane, R. A., Kane, R. L., \& Finch, M. D. (1995). Who cares? The size, scope, and composition of the caregiver support system. The Gerontologist, 35, 489-497.

Peters-Davis, N. D., Moss, M. S., \& Pruchno, R. A. (1999). Children-inlaw in caregiving families. The Gerontologist, 39, 66-75.

Pruchno, R., Burant, C., \& Peters, N. (1997). Typologies of caregiving families: Family congruence and individual well-being. The Gerontologist, 37, 157-167.

Pruchno, R., \& Postashnik, S. (1998). Caregiving spouses: Physical and mental health in perspective. Journal of the American Geriatrics Society, 37, 697-705.

Rodriguez, J., Kosloski, K., \& Montgomery, R. (2003). Evaluating a practice-oriented service model to increase the use of respite services among minorities and rural caregivers. The Gerontologist, 43, 916-924.

SAS Institute. (1999). SAS 8.0 [Computer software]. Cary, NC: SAS Institute.

Scharlach, A., Kellam, R., Ong, N., Goldstein, C., \& Baskin, A. (2006). Cultural attitudes and caregiver service use: Lessons from focus groups with racially and ethnically diverse family caregivers. Journal of Gerontological Social Work, 47(1/2), 133-156.

Schulz, R., O’Brien, A., Bookwala, J., \& Fleissner, K. (1995). Psychiatric and physical morbidity effects of dementia caregiving: Prevalence, correlates, and causes. The Gerontologist, 35, 771-791.

Schulz, R., \& Williamson, G. (1991). A two-year longitudinal study of depression among Alzheimer's caregivers. Psychology and Aging, 6, 569-578.

Semple, S. J. (1992). Conflict in Alzheimer's caregiving families: Its dimensions and consequences. The Gerontologist, 32, 648-655.

Smerglia, V., \& Deimling, G. (1997). Care related decision making satisfaction and caregiver well-being. The Gerontologist, 37, 658-665.

Sobel, M. E. (1982). Asymptotic confidence intervals for indirect effects in structural equation models. In S. Leinhardt (Ed.), Sociological methodology (pp. 290-312). Washington, DC: American Sociological Association.

Sobel, M. E. (1986). Some new results on indirect effects and their standard errors in covariance structure models. In N. Tuma (Ed.), Sociological methodology (pp. 159-186). Washington, DC: American Sociological Association.

Strawbridge, W. J., \& Wallhagen, M. I. (1991). Impact of family conflict on adult child caregivers. The Gerontologist, 31, 770-771.

Tennstedt, S. L., McKinlay, J. B., \& Sullivan, L. M. (1989). Informal care for frail elders: The role of secondary caregivers. The Gerontologist, 29, 677-683.

Weihs, K., Fisher, L., \& Baird, M. (2002). Families, health, and behavior. A section of the commissioned report by the committee on health and behavior: Research, practice, and policy. Families, Systems, and Health, 20, 7-46.

Yee, J., \& Schulz, R. (2000). Gender differences in psychiatric morbidity among family caregivers: A review and analysis. The Gerontologist, 40, $147-164$. 